\title{
FACTORES SOCIOECONÓMICOS COADYUDANTES EN PERITONITIS AGUDA HOSPITAL DE APOYO "HIPÓLITO UNANUE" TACNA PRIMER SEMESTRE 2002
}

Salvador Moarri Hoss', Luz Portugal Gallegos ${ }^{2}$, Roberto Pinto Morroy $^{3}$; Rina Alvarez Becerra ${ }^{3}$

\author{
R E S U MEN
}

El objetivo de la investigación fue determinar los factores socioeconómicos coadyuvantes en peritonitis aguda en el Hospital Hipólito Unanue de Tacna.

Mediante un estudio prospectivo de corte longitudinal, se ha recolectado los datos directamente en 108 pacientes portadores de apendicitis aguda, por ser la patologia màs frecuente de abdomen agudo y en consecuencia en la que se dá el mayor número de complicaciones.

De los 108 casos de apendicitis aguda diagnosticados y operados, se encontró que el grupo etáreo de sexo masculino fue màs afectado el de varones $(62,04 \%)$, y el $38.89 \%$ se encontraba entre los 20 y 29 años de edad. En relación a la procedencia el $44.4 \%$ procedía del cercado. En lo referente al tipo de alimentaciòn es catalogada como regular en 74,07\% . La vivienda es propia en $79.63 \%$.

En lo concerniente a los signos y sintomas de la apendicitis aguda, los encontrados concuerdan con lo referido a la literatura.

Fueron diagnosticados como apendicitis aguda complicada 54 casos (50\%).

Los antibióticos utilizados fueron los de tipo genérico, por su menor costo y ser más asequibles a la economía de los pacientes.

\section{$A B S T R A C T$}

The objective of the investigation was determine the socioeconomic factors that influence on sharp peritonitis in Hipolito Unanue Hospital of Tacna.

The data has been gathered directly in 108 patient, whossufer from sharp appendicitis, by means of a prospective study of longitudinal cut. Due to the fact that it is most frequent pathology (sharp abdomen) and as a consequence it is one that gives the biggest number of complications.

Of the 108 diagnosed cases of sharp appendicitis operated, It was found that the most etareo group was of males (62,04\%), and $38.89 \%$ was between the 20 and 29 years of age. In relation to the origin $44.4 \%$ came from the city center. Regardind to the food type it is classified as regular in $74.07 \%$ the housin is own in $79.63 \%$.

Concerning to signs and symtoms of sharp appendicitis the ones found agree with those referred in medical literature.

They were diagnosed as sharp appendicitis in 54 cases ( 50\%).

The used antibiotics were those of generic type, because of their smallest cost being in this way affordable to the patients economy.

\section{INTRODUCCIÓN}

El proceso peritónico agudo, ha aumentado en los ultimos años, razón por la cual, nos ha motivado hacer un estudio de los factores socioeconómicos que influyeron de manera indirecta, en esta complicación, que de por sí, se considera grave, puesto que en muchos casos puede conducir a la muerte.
Resulta en la actualidad, que por diversos motivos, los pacientes acuden tardiamente, con la complicación peritonitica aguda, ocasionando una serie do inconvenientes tanto para el enfermo como para la Institución de Salud.

El trabajo se realizó en el Hospital de Apoyo "Hipólito Unanue" de Tacna, en el Departamento de Cirugía, Sección de Hospitalización.

(1) (2)(3) Médico

(4) Magister en Docencia Universitaria 


\section{MATERIAL Y MÉTODOS}

\section{TIPO DE ESTUDIO}

Es descriptivo, prospectivo de corte longitudinal.

Los datos se obtuvieron discretamente, proporcionados por cada paciente.

\section{POBLACIÓN Y MUESTRA}

La población es referida a todos los pacientes con procesos quirúrgicos abdominales, hospitalizados en el primer semestre del 2002.

\section{VARIABLES DE ESTUDIO}

\section{Variable independiente:}

Paciente con peritonitis aguda, como aplicación de proceso inicial generalmente de causa infecciosa.

\section{Variables dependientes:}

Edad, sexo, ocupación, procedencia, las referentes a las condiciones socioeconómicas y las consideradas en el aspecto clínico del estudio.

\section{RECOLECCIÓN DE DATOS}

Se hizo a través de un instrumento pre elaborado con variables operacionalizados los datos son fácilmente obtenidos, ya que la ficha se confeccionó de manera simple y manejable.

\section{RESULTADOS Y DISCUSIÓN}

Consideramos los siguientes puntos:
1) Aspectos Generales
2) Condiciones Socioeconómicas
3) Aspectos Clínicos

\section{ASPECTOS GENERALES}

1.1. Distribución de pacientes por edad (cuadro №1)

Según la encuensta realizada, obtenemos como dato importante que el mayor número de pacientes corresponde al grupo etáreo comprsnidido entre los 20 y 29 años, con un porcentaje de $38.8 \% \%$

Luego sigue la frecuencia el grupo etarteo entre $30 y$ 39 años, con un procentaje de $13.52 \%$, edad considerada también de plena actividad laboral.

Si nos referimos al sexo, según el cuadro $\mathrm{N}^{\circ} 01$ predomina el sexo masculino con 67 casos $(62.09 \%)$ sobre el sexo femenino con 41 enfermas $(37.96 \%)$.

\subsection{Distribución de pacientes por sexo (cuadro $\mathrm{N}^{\circ} 02$ )}

Para tener una visión general en este parámetro, tenemos el cuadro $\mathrm{N}^{\circ} 02$, donde 67 casos son de sexo masculino y 41 de sexo femenino. Objetivamente apreciamos la proporción en el gráfico $\mathrm{N}^{\circ} 02$ (62\% sexo masculino, $38 \%$ sexo femenino).

1.3. Distribución de pacientes según ocupación (cuadro $\mathrm{N}^{\circ} 03$ )

Respecto a la ocupación notamos, según el cuadro $\mathrm{N}^{\circ} 03$, que el mayor número de casos está constituido por estudiantes, con un total de 26 casos, lo que hace un $28,70 \%$, siguiéndole en frecuencia, personas que se dedican a labores de casa con 26 pacientes, dando un procentaje de $24.07 \%$, siguen en orden decreciente comerciantes con 11 casos $(10.19 \%)$; obreros 10 pacientes (0.5.26\%), empleados con 9 casos $(08.33 \%)$, chofer con 6 pacientes $(05.56 \%)$; finalmente se consideran en otras ocupaciones 15 enfermos, teniendo un porcentaje de $13,89 \%$.

1.4. Distribución de pacientes según su procedencia (cuadro $\mathrm{N}^{\circ} \mathrm{O}$ )

Observainos según en cuadro $\mathrm{N}^{\circ} 4$ que la mayor frecuencia corresponde a pacientes que habitan en el cercado de la ciudad de Tacna, con un total de 48 pacientes, obteniéndose un $44,44 \%$, a estos pacientes les es fácil acudir al Hospital de Apoyo "Hipólito Unanue", ya que se encuentra ubicado en el cercado.

Sigue en frecuencia con 24 enfermos los procedentes de Gregorio Albarracin haciendo un $22.22 \%$.

En forma decreciente continuán los pacientes procedentes de Ciudad Nuevacon $11.11 \%$ luego del Alto de la Alianza con $08.33 \%$.

De otros lugares la frecuencia es minima, totalizando 15 casos, lo cual nos da un porcentaje de $13.88 \%$.

En el gráíco $\mathrm{N}^{\circ} 4$, notamos la predominancia de pacientes procedentes del cercado, seguido de Gregorio Albarracín, Ciudad Nueva y Alto de la Alianza. De los demás lugares, como se ve, están representados por barras de pequeña longitud.

\section{CONDICIONES SOCIOECONÓMICAS}

Este aspecto lo consideramos el más importante, ya que el motivo de nuestro trabajo, está dirigido a darle valor a la situación social y económica del paciente. Mayores gastos tanto al paciente como al Instituto de Salud que recepciona al paciente para su tratamiento. 


\subsection{Condiciones de trabajo (cuadro $\mathrm{N}^{\circ} 05$ )}

Sólo 51 pacientes de los 108 estudiados trabajan, lo que hace un porcentaje de $47.22 \%, 57$ pacientes no trabajan, es decir un $52.78 \%$ de casos que están en esta condición desfavorable.

\subsection{Condición de la vivienda (cuadro $\mathrm{N}^{\circ} 06$ )}

Analizando el cuadro $\mathrm{N}^{\circ} 05$ vemos que 86 pacientes tienen vivienda propia $(79.63 \%)$. En cambio 22 pacientes habitan en casa alquilada, es decir sólo el $20.37 \%$.

\subsection{Condiciones higiénicas (cuadro $\mathrm{N}^{\circ} 07$ )}

Consideramos tres categorias: Buena, Regular y Mala; nuestro trabajo no pretende investigar los detalles respecto a las condiciones higiénicas.

Si observamos el cuadro $N^{\circ} 07$, vemos que según declaración de los pacientes, 44 viven en buenas condiciones higiénicas, lo que hace un $40.74 \%$, la mayoría de pacientes, es decir 59 de ellos, declara que vive en condiciones higienicas regulares, es decir $54.63 \%$ y 5 pacientes refieren vivir en malas condiciones higiénicas (4.63).

\subsection{Servicios con que cuenta la vivienda (cuadro $\mathrm{N}^{\circ} 08$ )}

Consideramos las viviendas si cuentan con servicios de agua y desague, que de alguna forma influyen en las condiciones higiénicas.

Apreciamos que los 108 pacientes cuentan con servicios de luz, es decir el $100 \%$.

Sin servicio de agua potable, y según este cuadro figuran 04 enfermos que no tienen este servicio indispensable, obteniendo un porcentaje de $3.70 \%$. Igualmente, carecen de desague 06 pacientes $(5.55 \%)$.

\subsection{Tipo de alimentación (cuadro $N^{\circ}$ 09)}

Si se consideran a 27 pacientes con buen tipo de alimentación, lo que representa un $25 \%$ parte, con tipo de alimentación regular 80 pacientes, $(74.07 \%)$ es decir casi las $3 / 4$ partes de los casos estudiados, un paciente $(0.93 \%)$ cuya alimentación es mala.

\section{ASPECTOS CLINICOS}

\subsection{Tiempo de enfermedad (cuadro $N^{\circ} 10$ )}

50 pacientes, de los 108 estudiados tuvieron un dia de enfermedad, es decir el $46.30 \%$, le sigue en frecuencia con 2 dias de tiempo de enfermedad 25 pacientes, con $23.15 \%$ de incidencia, luego tenemos 16 pacientes con 3 dias de enfermedad antes de la consulta, es decir $14.81 \%$.

El resto de pacientes, que suman 17 tuvieron 40 más días (15.73\%).

\subsection{Sintomas principales (cuadro $N^{0} 11$ )}

El $100 \%$ de pacientes, o sea los 108 casos estudiados presentaron náuseas y vómitos, 06 pacientes diarrea, sintoma infrecuente en peritonitis aguda; 44 pacientes tuvieron sensación de alza térmica, 05 pacientes presentaron balonamiento abdominal y 02 pacientes falta de eliminación de heces y gases.

\subsection{Signos principales(cuadro $N^{\circ} 12$ )}

$100 \%$ de pacientes estudiados, es decir los 108 enfermos presentaron dolor objetivo a la palpación de fosa iliaca derecha; otros signos: ruidos hidroaereos disminuidos (sólo un paciente lo presento) y timpanismo.

\subsection{Diagnóstico pre-operatorio (cuadro $N^{0} 13$ )}

Por apendicitis aguda simple, sumando 54 enfermos, lo que hace un $50 \%$ siendo 32 de ellos de sexo masculino $(29.63 \%)$ y 22 pacientes de sexo femenino $(20.33 \%)$. Otros presentaron Apendicitis Aguda Complicada (2.78\%).Plastron Abscesazo (1.85\%).

\subsection{Intervención quirúrgica realizada (cuadro $\mathrm{N}^{\circ}$ 14)}

Apendicectomía a 58 enfermos, es decir al $53.70 \%$, se efectuó apendicectomia más drenaje, a 39 pacientes, $(36.11 \%)$. Laparatomia exploradora a 11 pacientes, es decir al $10.19 \%$.

\subsection{Complicaciones post-operatorlas} (cuadro $\mathrm{N}^{\circ} 15$ )

21 pacientes con complicaciones post operatorias, de los 108 enfermos (19.44\%) Mencionamos: infección de herida operatoia 18 pacientes, 01 paciente con fistula estercorácea. 01 con absceso subaponeurótico un paciente hizo absceso residual $(4.76 \%)$.

\subsection{Antibióticos utilizados en el Post-operatorio (cuadro $\mathrm{N}^{\circ}$ 16)}

Se usaron en orden de frecuencia: cloroanfenico $(34.15 \%)$ Gentamicina $(22.93 \%)$ Ceftriaxona, $(19.51 \%)$ $(7.32 \%)$. cefalotina sea en un $(10.73 \%)$, la cefalotine $(10.73 \%)$ Otros: Amikacina, Metronidazol, Osmoflox Cefuroxina. 
CUADRO N 01

DISTRIBUCIÓN DE PACIENTES CON PERITONITIS AGUDA SEGÚN GRUPO ETÁREO

\begin{tabular}{|c|c|c|c|c|c|c|}
\hline \multirow{2}{*}{$\begin{array}{r}\text { GRXPQ } \\
\text { ETARTO }\end{array}$} & \multicolumn{2}{|c|}{ MASCULINO } & \multicolumn{2}{|c|}{ FEM RN INO } & \multicolumn{2}{|c|}{ TOTAL } \\
\hline & $\mathrm{N}^{6}$ & $\%$ & $\mathrm{~N}^{\circ}$ & $\%$ & $\mathrm{~N}^{2} \mathrm{i}$ & $\%$ \\
\hline $\begin{array}{r}10-19 \\
20-29 \\
\end{array}$ & $\begin{array}{l}16 \\
21 \\
\end{array}$ & $\begin{array}{l}14.81 \\
19.44 \\
\end{array}$ & $\begin{array}{l}10 \\
21\end{array}$ & $\begin{array}{c}9.26 \\
19.44 \\
\end{array}$ & $\begin{array}{l}26 \\
42\end{array}$ & $\begin{array}{l}24.07 \\
38.89\end{array}$ \\
\hline $30-39$ & 14 & 12.96 & 6 & 5.56 & 20 & 15.82 \\
\hline $\begin{array}{l}40-49 \\
50-59 \\
60-69 \\
\end{array}$ & $\begin{array}{l}08 \\
01 \\
06 \\
\end{array}$ & $\begin{array}{l}7.41 \\
0.93 \\
5.56 \\
\end{array}$ & $\begin{array}{l}2 \\
2 \\
\end{array}$ & $\begin{array}{l}1.85 \\
0.00 \\
1.85 \\
\end{array}$ & $\begin{array}{l}10 \\
01 \\
08 \\
\end{array}$ & $\begin{array}{l}09.26 \\
00.93 \\
07.41 \\
\end{array}$ \\
\hline 70 A MÁs & 01 & 0.93 & - & 0.00 & 01 & 00.93 \\
\hline TOTAL & 67 & 62.04 & 41 & 37.96 & 106 & 100.00 \\
\hline
\end{tabular}

FUENTE ENCUESTA

\section{CUADRO $\mathrm{N}^{\circ} 02$}

DISTRIBUCIÓN DE PACIENTES CON PERITONITIS AGUDA SEGÚN SEXO

\begin{tabular}{|c|c|c|c|c|c|}
\hline \multicolumn{2}{|c|}{ AMASCULNO } & \multicolumn{2}{|c|}{ Hrymara } & \multicolumn{2}{c|}{ TOTAL } \\
\hline $\mathrm{N}^{\circ}$ & $\%$ & $\mathrm{~N}^{\circ}$ & $\%$ & $\mathrm{~N}^{\circ}$ & $\%$ \\
\hline 67 & 62.04 & 41 & 37.96 & 106 & 100.00 \\
\hline
\end{tabular}

FUENTE ENCUESTA

\section{CUADRO N ${ }^{\circ} 03$}

DISTRIBUCIÓN DE PACIENTES CON PERITONITIS AGUDA SEGÚN OCUPACIÓN

\begin{tabular}{|c|c|c|c|c|c|c|}
\hline \multirow{2}{*}{ ocupación } & \multicolumn{2}{|c|}{ MAscuigyo } & \multicolumn{2}{|c|}{ FaYrange } & \multicolumn{2}{|c|}{ mogu } \\
\hline & $N^{o}$ & $\%$ & $\mathbf{N}^{\circ}$ & $\%$ & $N^{2}$ & $\%$ \\
\hline OBRERO & 9 & 8.33 & 1 & 0.93 & 10 & 09.26 \\
\hline EMPLEADO & 9 & 8.33 & . & 0.00 & 9 & 08.33 \\
\hline SU CASA & 4 & 3.70 & 22 & 20.37 & 26 & 24.07 \\
\hline ESTUDIANTE & 10 & 17.59 & 12 & 11.11 & 31 & 28.70 \\
\hline COMERCIANTE & 8 & 7.41 & 3 & 2.78 & 11 & 10.19 \\
\hline CHOFER & 6 & 5,56 & - & 0.00 & 6 & 05.56 \\
\hline OTROS & 12 & 11.11 & 3 & 2.76 & 15 & 13.89 \\
\hline TOTAL & 67 & 62.04 & 41 & 37.96 & 108 & 100.00 \\
\hline
\end{tabular}

FUENTE ENCUESTA
CUADRO $\mathrm{N}^{\circ} 04$

DISTRIBUCIÓN DE PACIENTES CON PERITONITIS AGUDA SEGUUN PROCEDENCIA

\begin{tabular}{|c|c|c|c|c|c|c|}
\hline PRocfphods & Mise & $\begin{array}{r}\text { Eutyo } \\
\% \\
\%\end{array}$ & 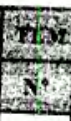 & $\begin{array}{l}\text { exing } \\
\% \\
\%\end{array}$ & $\frac{1}{x^{2}}$ & 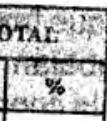 \\
\hline CERCADO & 32 & 29.83 & 161 & 14.1 & 46 & 44.44 \\
\hline $\begin{array}{l}\text { ALTO DE LA } \\
\text { ALIANZA }\end{array}$ & 6 & 5.56 & 3 & 2.76 & 9 & 06.33 \\
\hline CIUDAD NUEVA & 8 & 7.41 & 4 & 3.70 & 12 & 11.11 \\
\hline BOLOGNESI & 1 & 0.93 & 2 & 1.85 & 3 & 02,78 \\
\hline HÁBITAT & 1 & 0.93 & - & 0.00 & 1 & 00.93 \\
\hline NATIVIDAD & 2 & 1.85 & - & 0.00 & 2 & 01.85 \\
\hline CALANA & 1 & 0.93 & - & 0.00 & 1 & 00.93 \\
\hline LA YARADA & - & 0.00 & I & 0.93 & 1 & 00.93 \\
\hline ITE & . & 0.00 & 1 & 0.93 & 1 & 0.93 \\
\hline $\begin{array}{l}\text { GREGORIO } \\
\text { ALBARRACIN }\end{array}$ & 12 & 11.11 & 12 & 11.11 & 24 & 22.22 \\
\hline POCOLLAY & - & 0.00 & 1 & 0.93 & 1 & 00.93 \\
\hline CANDARAVE & 1 & 0.93 & - & 0.00 & 1 & 00.93 \\
\hline TTCACO & 1 & 0.93 & - & 0.00 & 1 & 00.93 \\
\hline ILABAYA & 1 & 0.93 & - & 0.00 & 1 & 00.93 \\
\hline CAÑETE & 1 & 0.93 & - & 0,00 & 1 & 00.93 \\
\hline CHILE & - & 0.00 & 1 & 0,93 & 1 & 00.93 \\
\hline TOTAL & 67 & 62.04 & 41 & 37.96 & 108 & 100.00 \\
\hline
\end{tabular}

FUENTE ENCUESTA

\section{CUADRO $N^{\circ} 05$}

DISTRIBUCION DE PACIENTES CON PERITONITIS AGUDA SEGÚN CONDICIÓN DE TRABAJO

\begin{tabular}{|c|c|c|c|c|c|c|}
\hline \multirow{2}{*}{$\begin{array}{r}\text { VIVIENDA } \\
\end{array}$} & \multicolumn{2}{|c|}{ MASCULIVO } & \multicolumn{4}{|c|}{ 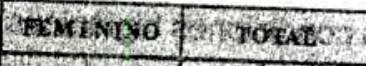 } \\
\hline & N: & get & Nie: & 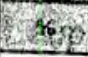 & zas? & \% \\
\hline BUENA & 18 & 16.67 & 9 & 08.33 & 27 & 25.00 \\
\hline REGULAR & 49 & 45.37 & 31 & 28.70 & 80 & 74.07 \\
\hline MALA & - & - & 1 & 0.93 & 1 & 0.93 \\
\hline TOTAL & 67 & 62.04 & 41 & 37.96 & 108 & 100.00 \\
\hline
\end{tabular}

FUENTE ENCUESTA

\section{CUADRO $N^{\circ} 06$}

DISTRIBUCIÓN DE PACIENTES CON PERITONITIS AGUDA SEGÚN CONDICIÓN DE LA VIVIENDA

\begin{tabular}{|c|c|c|c|c|c|c|}
\hline \multirow{2}{*}{ 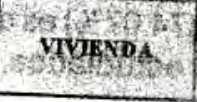 } & \multicolumn{2}{|c|}{ Arspeveuno } & \multicolumn{2}{|c|}{ Tox $1 \times 150$} & \multicolumn{2}{|c|}{ 6 froorkers? } \\
\hline & $x^{2}$ का & 98 & 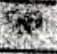 & $5 \%$ & x. & मूet \\
\hline PROPIA & 53 & 49.07 & 33 & 30.56 & 86 & 79.63 \\
\hline ALQUILADA & 14 & 12.96 & 08 & 07.41 & 22 & 20.37 \\
\hline TOTAL & 67 & 62.04 & 41 & 37.96 & 108 & 10.00 \\
\hline
\end{tabular}

FUENTE ENCUESTA 
CUADRO N 07

DISTRIBUCIÓN DE PACIENTES CON PERITONITIS AGUDA SEGÚN CONDICIONES HIGIENICAS

\begin{tabular}{|c|c|c|c|c|c|c|}
\hline \multirow{2}{*}{ VIVIENDA } & \multicolumn{2}{|c|}{ MASCHIIO } & \multicolumn{2}{|c|}{ 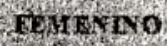 } & \multicolumn{2}{|c|}{ TotaI } \\
\hline & $N^{0}$ & $\%$ & $\mathrm{~N}^{\circ}$ & $\%$ & $x^{2}$ & $\%$ \\
\hline BUENA & 27 & 25.00 & 17 & 15.74 & 44 & 40.74 \\
\hline REGULAR & 37 & 34.26 & 22 & 20.37 & 59 & 54.63 \\
\hline MALA & 03 & 02.78 & 02 & 01.85 & 05 & 04.63 \\
\hline TOTAL & 67 & 62.04 & 41 & 37.96 & 108 & 100.00 \\
\hline
\end{tabular}

FUENTE ENCUESTA

\section{CUADRO $\mathrm{N}^{\circ} 08$}

DISTRIBUCIÓN DE PACIENTES CON PERITONITIS AGUDA SEGÚN SERVICIOS

\begin{tabular}{|c|c|c|c|c|c|c|c|c|c|c|c|c|}
\hline \multirow{3}{*}{ sermáas } & \multicolumn{4}{|c|}{ Mscauro } & \multicolumn{4}{|c|}{ revgeno } & \multicolumn{4}{|c|}{ WOAL } \\
\hline & \multicolumn{2}{|c|}{ s } & \multicolumn{2}{|c|}{ No } & \multicolumn{2}{|c|}{ fi } & \multicolumn{2}{|c|}{80} & \multicolumn{2}{|c|}{ s } & \multicolumn{2}{|c|}{ No } \\
\hline & $x$ & $\%$ & S. & $\%$ & (8) & $\%$ & * & $\%$ & A) & $\%$ & $x$ & \\
\hline $\mathrm{AgCu}$ & 60 & 55.55 & 3 & 278 & 44 & 40.74 & & 0.93 & 104 & 9230 & 4 & \\
\hline$A Q$ & 66 & 51.85 & 4 & 3.70 & 46 & 4259 & & 1.85 & 102 & 94.44 & 6 & \\
\hline z & 67 & 6203 & 0 & 0.00 & 41 & 37.96 & & 0.00 & 108 & 10000 & 0 & \\
\hline
\end{tabular}

FUENTE ENCUESTA

CUADRO $\mathrm{N}^{\circ} 09$

DISTRIBUCIÓN DE PACIENTES CON PERITONITIS AGUDA SEGÚN ALIMENTACIÓN

\begin{tabular}{|c|c|c|c|c|c|c|}
\hline \multirow{2}{*}{ conplatoN } & \multicolumn{2}{|c|}{ MASCU ANO } & \multicolumn{2}{|c|}{ Hovenpio } & \multicolumn{2}{|c|}{ Total } \\
\hline & Ni: & $\%$ & $\times 8$ & $\%$ & $x^{\circ}$ & $\%$ \\
\hline TRABAJA & 44 & 40.74 & 7 & 6.43 & 51 & 47.22 \\
\hline NO TRABAJA & 23 & 21.30 & 34 & 31.48 & 57 & 52.78 \\
\hline TOTAL & 67 & 62.04 & 41 & 37.96 & 108 & 100.00 \\
\hline
\end{tabular}

FUENTE ENCUESTA

CUADRO $\mathrm{N}^{\circ} 10$

DISTRIBUCIÓN DE PACIENTES CON PERITONITIS AGUDA SEGÚN TIEMPO DE ENFERMEDAD

\begin{tabular}{|c|c|c|c|c|c|c|}
\hline \multirow{2}{*}{$\begin{array}{l}\text { TEMPO DZ } \\
\text { ENFERMEISAD }\end{array}$} & \multicolumn{2}{|c|}{ Mascin no } & \multicolumn{2}{|c|}{ TMENDo } & \multicolumn{2}{|c|}{ rotal } \\
\hline & $\mathrm{N}^{0} \mathrm{C}$ & $\%$ & $\mathrm{No}^{6}$ & $\%$ & $\mathrm{x}^{2}$ & $\%$ \\
\hline $1 \mathrm{DIA}$ & 32 & 29.63 & 18 & 16.67 & 50 & 46.30 \\
\hline 2 DIAS & 12 & 11.11 & 13 & 12.04 & 25 & 23.15 \\
\hline 3 DIAS & 9 & 8.33 & 7 & 6.48 & 16 & 14.61 \\
\hline 4 DIAS & 3 & 2.78 & 1 & 0.93 & 4 & 03.70 \\
\hline 5 DIAS & 3 & 2.78 & 1 & 0.93 & 4 & 03.70 \\
\hline 6 DIAS & 2 & 1.85 & - & 0.00 & 2 & 01.85 \\
\hline 7 DIAS & 4 & 3.70 & 1 & 0.93 & 5 & 04.63 \\
\hline MAS 7 DIAS & 2 & 1.85 & - & 0.00 & 2 & 01.65 \\
\hline TOTAL & 67 & 62.04 & 41 & 37.96 & 100 & 100.00 \\
\hline
\end{tabular}

FUENTE ENCUESTA
CUADRO $\mathrm{N}^{\circ} 11$

DISTRIBUCIÓN DE PACIENTES CON PERITONITIS AGUDA SEGÚN SINTOMAS PRINCIPALES

\begin{tabular}{|l|c|}
\hline \multicolumn{1}{|c|}{ SHTTOMAS } & TOLAE \\
\hline DOLOR ABDOMINAL & 108 \\
\hline NÁUSEAS Y VÓMITOS & 60 \\
\hline DIARREAS & 06 \\
\hline SENSACIÓN TÉRMICA & 44 \\
\hline BALONAMIENTO ABDOMINAL & 05 \\
\hline FALTA DE ELIMINACIÓN DE HECES Y GASES & 02 \\
\hline
\end{tabular}

FUENTE ENCUESTA

CUADRO N ${ }^{\circ} 12$

DISTRIBUCIÓN DE PACIENTES CON PERITONITIS AGUDA SEGÚN SIGNOS

\begin{tabular}{|l|c|}
\hline \multicolumn{1}{|c|}{ SWITOMAS } & TOTAE \\
\hline DOLOR OBJEIIVO FID & 108 \\
\hline DOLOR A LA DESCOMPRENSIÓN & 60 \\
\hline RHA DISMINUIDOS & 06 \\
\hline TIMPANISMO & 44 \\
\hline
\end{tabular}

FUENTE ENCUESTA

CUADRO N ${ }^{\circ} 13$

DISTRIBUCIÓN DE PACIENTES CON PERITONITIS AGUDA SEGÚN DIAGNOSTICO PRE OPERATORIO

\begin{tabular}{|c|c|c|c|c|c|c|}
\hline \multirow{2}{*}{ 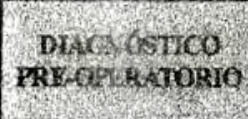 } & \multicolumn{2}{|c|}{ MAsGe } & \multicolumn{2}{|c|}{ panratios } & \multicolumn{2}{|c|}{ POTL } \\
\hline & $N^{6}$ & $\%$ & $x^{\circ}$ & 9 & Ne & $\%$ \\
\hline $\begin{array}{l}\text { Apendicitis aguda } \\
\text { simple }\end{array}$ & 32 & 29.63 & 22 & 20.37 & 54 & 50.00 \\
\hline $\begin{array}{l}\text { Apendicitis aguda } \\
\text { complicada por lec }\end{array}$ & 31 & 26.70 & 18 & 16.67 & 49 & 45.37 \\
\hline $\begin{array}{l}\text { Apendicitis aguda } \\
\text { complicada per. gral. }\end{array}$ & 2 & 1.85 & 1 & 0.93 & 3 & 2.78 \\
\hline Plastron abscedado & 2 & 1.85 & - & 0.00 & 2 & 1.85 \\
\hline Total & 67 & 62.04 & 41 & 37.96 & 108 & 100.00 \\
\hline
\end{tabular}

FUENTE ENCUESTA

CUADRO No 14

DISTRIBUCIÓN DE PACIENTES CON PERITONITIS AGUDA SEGÚN OPERACIÓN REALIZADA

\begin{tabular}{|c|c|c|c|c|c|c|}
\hline \multirow{2}{*}{ 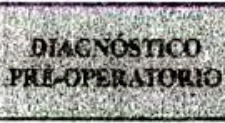 } & \multicolumn{2}{|c|}{ MaseñNo } & \multicolumn{2}{|c|}{ Fovisuro } & \multicolumn{2}{|c|}{ mojal. } \\
\hline & $x^{2}$ & $\%$ & $x^{2}$ & $\%$ & No & $\%$ \\
\hline APENDICECTOMIA & 37 & 34.26 & 21 & 19.44 & 58 & 53.10 \\
\hline $\begin{array}{l}\text { APENDICECTOMIA + } \\
\text { DRENAJE }\end{array}$ & 21 & 19.44 & 18 & 16.67 & 39 & 36.11 \\
\hline $\begin{array}{l}\text { LAPAROTOMIA } \\
\text { EXPLORATORIA }\end{array}$ & 9 & 8.33 & 2 & 1.85 & 11 & 10.19 \\
\hline TOTAL & 67 & & 41 & 37.96 & 108 & 100.00 \\
\hline
\end{tabular}

FUENTE ENCUESTA 
CUADRO N ${ }^{\circ} 15$

DISTRIBUCIÓN DE PACIENTES CON PERITONITIS AGUDA SEGÚN COMPLICACIONES

\begin{tabular}{|c|c|c|c|c|c|c|}
\hline \multirow{2}{*}{ CoMmIICACIONES } & \multicolumn{2}{|c|}{ MAscuine } & \multicolumn{2}{|c|}{ Phino } & \multicolumn{2}{|c|}{ Tora } \\
\hline & $\mathrm{N}^{\circ}$ & $\%$ & $x^{\circ}$ & $4 \%$ & $\mathrm{AP}^{2}$ & $\%$ \\
\hline $\begin{array}{l}\text { INFECCIÓN DE } \\
\text { HERIDA }\end{array}$ & 12 & 57.14 & 6 & 28.57 & 18 & 85.71 \\
\hline $\begin{array}{l}\text { FISTULA } \\
\text { ESTERCORACEA }\end{array}$ & 1 & 4.78 & - & 0.00 & 1 & 4.76 \\
\hline $\begin{array}{l}\text { ABSCESO SUB } \\
\text { APONEURORICO }\end{array}$ & 1 & 4.76 & - & 0.00 & 1 & 4.76 \\
\hline $\begin{array}{l}\text { ABSCESO SUB } \\
\text { RESIDUAL }\end{array}$ & 1 & 4.76 & - & 000 & 1 & 4.76 \\
\hline TOTAL & 15 & 71.43 & 6 & 28.57 & 21 & 100.00 \\
\hline
\end{tabular}

FUENTE ENCUESTA

CUADRO No 16

DISTRIBUCIÓN DE PACIENTES CON PERITONITIS AGUDA SEGÚN ANTIBIÓTICOS UTILIZADOS

\begin{tabular}{|l|c|c|c|c|c|c|}
\hline & MASCUINO & FEMINWO & \multicolumn{2}{|c|}{ rOMAI } \\
\cline { 2 - 8 } & $\mathrm{N}$ & $\%$ & $\mathbf{N}$ & $\%$ & $\mathbf{N}$ & $\%$ \\
\hline GENTAMICINA & 30 & 14.63 & 17 & 8.29 & 47 & 22.93 \\
\hline CLORANFENICOL & 47 & 22.93 & 23 & 11.22 & 70 & 34.15 \\
\hline MEIRONIDAZOL & 7 & 3.41 & 4 & 1.95 & 11 & 5.37 \\
\hline CEFTRIAXONA & 25 & 12.00 & 15 & 7.32 & 40 & 19.51 \\
\hline AMIKACINA & 7 & 3.41 & 5 & 2.44 & 12 & 5.85 \\
\hline CEFALOTINA & 15 & 7.32 & 7 & 3.41 & 22 & 10.73 \\
\hline OSMOFLOX & 2 & 0.98 & - & 0.00 & 2 & 0.98 \\
\hline CEFUROXINA & 1 & 0.49 & - & 0.00 & 1 & 0.49 \\
\hline TOTAL & 134 & 65.37 & 71 & 34.63 & 205 & 10.00 \\
\hline
\end{tabular}

FUENTE ENCUESTA

\section{CONCLUSIONES}

1.- La demora en acudir a la consulta médica para ser diagnosticados $\mathrm{y}$ tratados por cuadros de apendicitis aguda en nuestro trabajo se hizo más evidente en personas de escasos recursos económicos y en las que son dependientes de éstas.

2.- Como consecuencia de la demora en acudir a la consulta médica la incidencia de pacientes diagnosticados cono apendicitis aguda con algún tipo de complicación fue alta.

3.- En el Perú más del $60 \%$ de la población vive en la pobreza o extrema pobreza lo que hace que el acceso a los servicios de salud sea difícil o aun imposible en algunos casos por la falta de recursos económicos y esto se refleja en la demora en acudir a centros de salud en forma oportuna.

\section{RECOMENDACIONES}

1.- Realizar campañas de educación sanitaria dando relieve a las caracteristicas principales del cuadro clinico de la apendicitis aguda y haciendo especial énfasis en la importancia de acudir en forma oportuna a los centros de salud para asi evitar complicaciones que incluso pueden llevar al deceso del paciente.

2.- Que se amplie el seguro integral de salud a las personas mayores de 18 años que acrediten escasos recursos económicos o que los comités de indigencia de los hospitales se hagan cargo de esta población y no traten de evadir esta responsabilidad a fin de lograr mayores recursos propios

3. Hacer campañas de educación sanitaria, insistiendo en las condiciones higiénicas, tanto en la casa como en lo referente a medida personales.

4. Facilitar el acceso de pacientes a la consulta extrema.

5. Realizar charlas educativas en colegios de secundaria.

6. Dar preferencia a los pacientes que tengan este cuadro clínico.

7. Tratar de acortar el tiempo de hospitalización del paciente.

8. Recomendar el empleo de antibióticos - solos o combinados - en el post-operatorio.

\section{REFERENCIA BIBLIOGRÁFICA}

1. ANDER-EGG, Ezequiel. Técnicas de Investigación Social $14^{\circ}$ Edición, Argentina 1980.

2. BACON, Harry E..; ROSS, Sturat T. Atlas de Técnica Operatoria.- 1997. Librería y Editorial Bernales, S.R.L.. Buenos Aires. Argentina.

3. BERKOW Robert M.D. ; TALBOTT H. John, M.D. Manual Merck de Diagnóstico y Terapéutica. Rahway, N.J. Sexta Edición en Español. Editorial Merck \& Sharp Dohme Researcha Laboratorios. 1988.

4. CORNEJO GARRIDO, Gerardo. Manual de Semiología Quirúrgica. Sección Publicaciones. Universidad de Chile. 1990. Santiago, Chile.

5. Diccionario de Ciencias Médicas. Dorland. Barcelona. Séptima edición. El Ateneo S.A. 1991

6. Diccionario Pschyrembel de Ginecología y Obstetricia. Walter de Gruyter. Berlin. Nueva York. Edición Original. 1998

7. GARCIA FERNANDEZ, Dora. Metodología del Trabajo de Investigación, Editorial Trillas, 1998.

8. GARDNER, Ernest; GRAY, Donald J,; O'RAHILLY, Ronan. Anatomía. Quinta Edición, 1992. Salvat editores S.A.. Barcelona España. 